\title{
La nouvelle classification des abeilles (Hymenoptera, Apoidea, Apiformes) ou la chute de l'abeille mellifère (Apis mellifera L.) de son piédestal
}

Par Denis MICHEZ *

Histoire naturelle des abeilles

"Les classifications ne sont pas de simples procédures de rangement dans un monde divisé en catégories évidentes. Les taxinomies sont des décisions que l'Homme impose à la naturedes théories sur les causes de l'ordre dans la nature - et la chronique des revirements taxinomiques opérés au cours de l'histoire donne l'idée la plus juste des révolutions conceptuelles intervenues dans la pensée humaine. La nature objective existe, mais c'est seulement à travers la structure de nos systèmes taxinomiques que nous pouvons converser avec elle" (Gould 1996). Dans cet article, je vais tenter d'illustrer que l'histoire de la classification des abeilles au sens large (i.e. Apoidea Apiformes) éclaire magistralement cette réflexion de Gould. Nous sommes en effet à l'aube d'une "révolution conceptuelle" dans l'étude des abeilles suite aux nouvelles classifications proposées par Danforth et al. (2006a, b).

Les premiers pas de l'apidologie et de la systématique des abeilles se résument à l'exploitation, l'élevage, l'observation et l'étude d'une seule espèce: l'abeille mellifère, Apis mellifera L. Cet "apidocentrisme" est bien légitime, peu d'insectes présentent un rapport aussi privilégié avec l'Homme que l'abeille mellifère. L'importance de l'abeille est bien sûr avant tout liée au produit même de la ruche. Depuis longtemps, de nombreuses sociétés utilisent le miel comme principale source de sucre. Une des premières représentations d'insecte réalisée par l'homme montre d'ailleurs une silhouette féminine récoltant du miel dans une crevasse, entourée d'abeilles sommairement représentées (Figure 1). Par ailleurs, l'observation du travail acharné des ouvrières a fasciné de nombreux curieux, intellectuels ou scientifiques. Aristote fut le premier à décrire l'organisation d'une ruche dans Histoire des animaux (d'Aguillar 2006). Ses observations furent pendant longtemps les seules bases des recherches entomologiques. Bien plus tard, au $17^{\text {ème }}$ siècle, l'abeille mellifère fut l'un des premiers insectes examinés et décrit au microscope par Frederigo Cesi (Figure 2). L'abeille mellifère est ainsi, dès le départ, au cœur de la conceptualisation de l'entomologie et a fortiori de l'apidologie.

* Laboratoire de Zoologie, Université de Mons-Hainaut, Av. Maistriau 19, B-7000 Mons, Belgique. E-mail: denis.michez@umh.ac.be

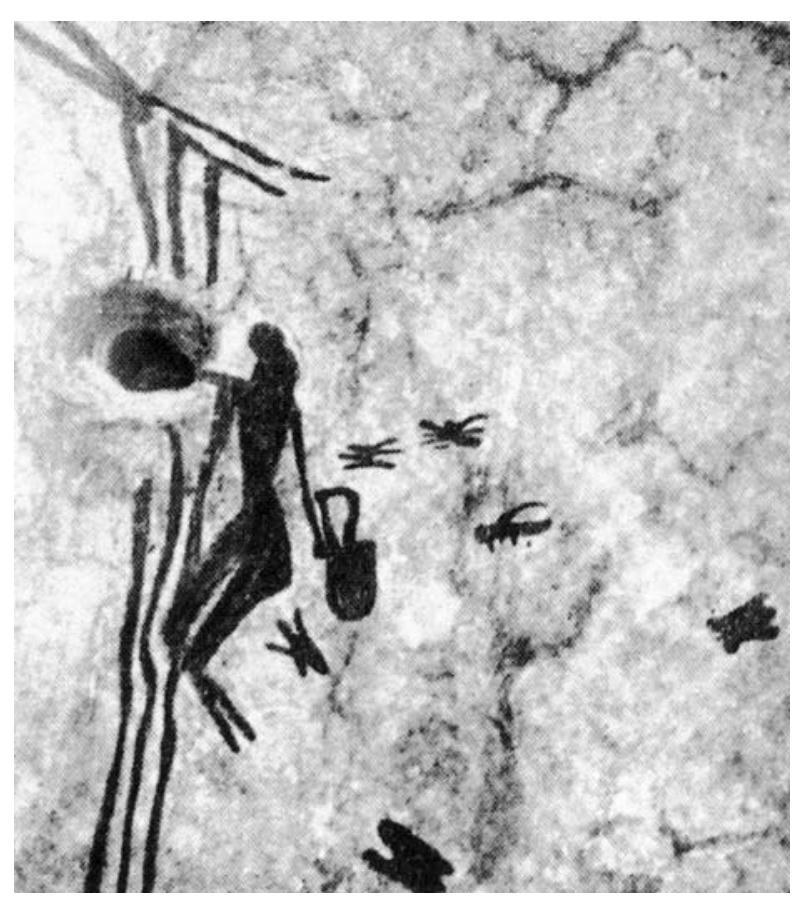

Figure 1. Peinture néolithique rupestre de la Cueva de la Araña (Valence, Espagne ; -5000 av. J.-C.) représentant la "cueillette" du miel par une silhouette féminine..

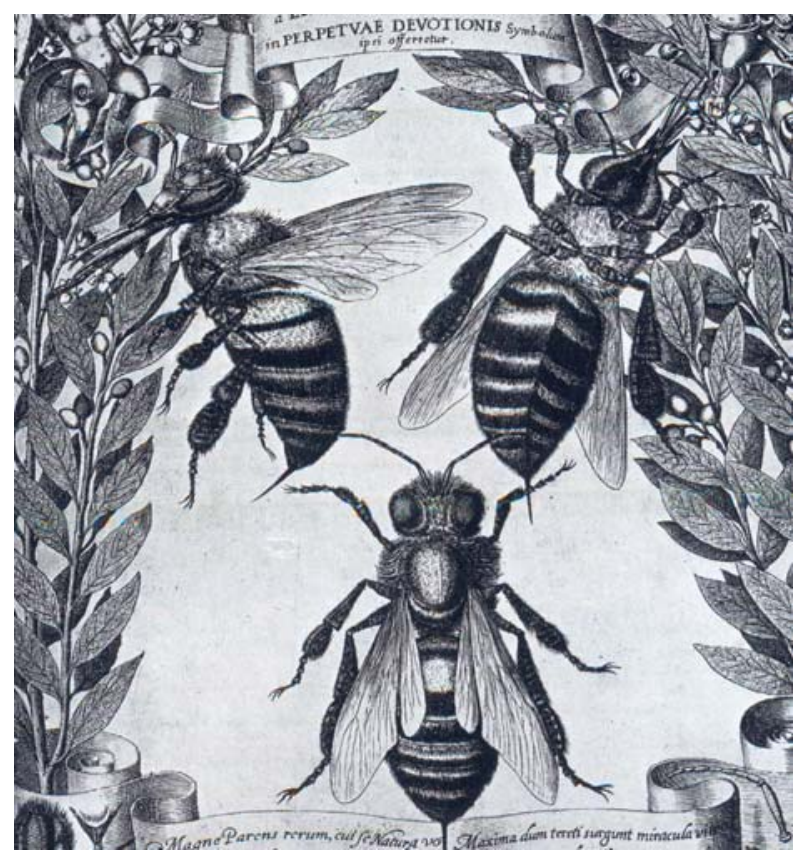

Figure 2. Frederigo Cesi (1625), Apiarium. Première figure d'Apis mellifera examinée sous microscope. 
Tableau 1. Classification des abeilles selon Latreille (1802)

\section{Famille des Andrenetes (Andrenetae)}

Division I

Division II

Famille des Apiaires (Apiariae)

Mégachiles
Nomades
Eucères
Podalitries
Clavicères
Xylocopes
Euglosses
Bourdons
Apiaires domestiques

Kirby (1802) et Latreille (1802) proposèrent presque simultanément une première classification globale pour les abeilles au sens large (ce que nous appelons maintenant les Apoidea Apiformes). Ils placèrent "logiquement" l'abeille mellifère au coeur de la classification. Kirby (1802) distingua deux genres, Apis L. et Melitta Kirby, qui reprennent respectivement les abeilles à langue longue et les abeilles à langue courte. Latreille (1802) proposa les deux mêmes groupes (nommés respectivement Apiarae et Andrenetae) en y ajoutant plusieurs subdivisions (Tableau 1). Au cours du $19^{\text {ème }}$ siècle, de nombreux nouveaux genres ont été décrits et les classifications se sont affinées. Parmi d'autres, Schenk (1860) et Thomson (1872) ont décrits de nombreuses nouvelles familles et tribus (Tableau 2; voir Michener (2000) et Engel (2005) pour plus d'informations).

$\mathrm{Au}$ cours de ces changements successifs de classification, la notion d'échelle des êtres (scala naturae) reste très présente, consciemment ou pas. Pour preuve ce passage du fameux ouvrage de Maurice Maeterlinck (prix Nobel de littérature), la vie des abeilles : “... toute sa vie [d'ouvrière d'abeille mellifère] est un sacrifice total à l'être innombrable et perpétuel dont elle fait
Langue arrondie (Colletes, Hylaeus)
Langue pointue (Andrena, Dasypoda)

\author{
(Megachile) \\ (Epeolus, Melecta, Nomada) \\ (Eucera) \\ $($ Centris , Podalirius $=$ Anthophora $)$ \\ $($ Clavicerna $=$ Ceratina $)$ \\ (Xylocopa) \\ (Euglossa) \\ (Bombus) \\ (Apis)
}

partie. Il est curieux de constater qu'il n'en fut pas toujours ainsi. On retrouve aujourd'hui, parmi les hyménoptères mellifères, tous les états de la civilisation progressive de notre abeille domestique. Au bas de l'échelle, elle travaille seule, dans la misère ; souvent elle ne voit même pas sa descendance: (les Prosopis, les Collètes, etc.), parfois même elle vit au milieu de l'étroite famille annuelle qu'elle crée (les Bourdons). Elle forme ensuite des associations temporaires (les Panurges, les Dasypodes, les Halictes, etc...), pour arriver enfin, de degrés en degrés, à la société à peu près parfaite mais impitoyable de nos ruches, où l'individu est entièrement absorbé par la république, et où l'individu est entièrement sacrifié à la cité abstraite et immortelle de l'avenir" (Maeterlinck 1901).

La première classification moderne des abeilles est présentée dans la thèse de doctorat de Charles D. Michener (Michener 1944). Dans cette classification phylogénétique, l'abeille mellifère occupe toujours le sommet de l'arbre (Figure 3). C'est d'ailleurs cette hypothèse qu'il retiendra au cours de toute sa brillante carrière synthétisée dans sa dernière grande oeuvre, Bees of the World, dont les deux dernières pages sont consacrées au genre Apis (Michener 2000, 2006).

Tableau 2. Classification des abeilles selon Schenk (1860)

\begin{tabular}{cl}
\hline Sous-famille Andrenidae & $($ Andrena, Colletes, Hyaleus $=$ Halictus, Nomia $)$ \\
Prosopidae & $($ Prosopis $=$ Hylaeus $)$ \\
Sphecodidae & (Sphecodes $)$ \\
Panurgidae & $($ Dasypoda, Dufourea, Panurgus $)$ \\
Rophitidae & $($ Halictoides, Rhophites $)$ \\
Melittidae & $($ Macropis, Melitta, Panurginus $)$ \\
Megachilidae & $($ Anthidium, Lithurgus, Megachile $)$ \\
Anthophoridae & $($ Anthophora, Ceratina, Eucera, Melitturga, Systropha) \\
Xylocopidae & $($ Xylocopa $)$ \\
Apidae & $($ Apis, Bombus $)$ \\
Psithyridae & $($ Psithyrus $)$ \\
Melectidae & $($ Epeolus, Melecta, Nomada, Pasitines $)$ \\
Stelidae & $($ Coelioxys, Dioxys, Stelis $)$
\end{tabular}




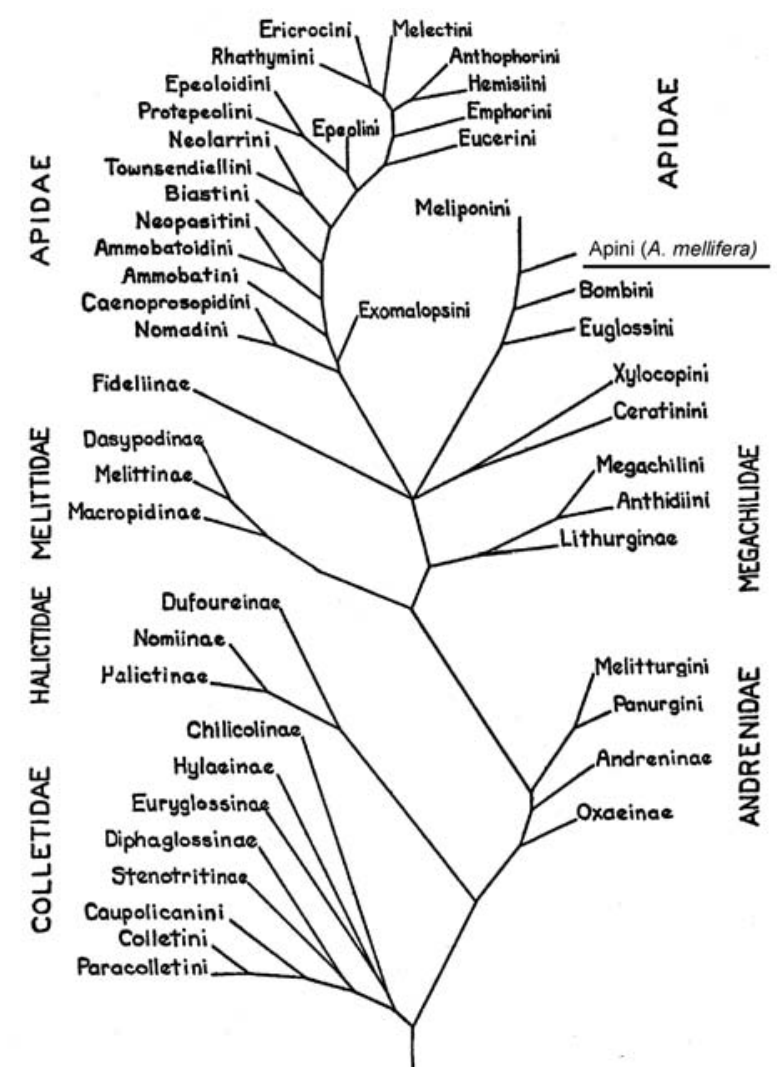

Figure 3. Phylogénie des Apoidea Apiformes basée sur la morphologie des adultes (d'après Michener 1944).

Le corollaire de cette hypothèse est la position basale de la famille des Colletidae. En référence à cette famille, l'abeille ancestrale posséderait donc une langue courte et bifide, serait apparue dans le Gondwana (paléocontinent regroupant Afrique, Amérique du Sud, Antarctique et Australie) et serait plutôt généraliste dans son mode d'alimentation.

Cependant, au cours de ces six dernières décennies dominées par le travail de Michener, d'autres hypothèses déstabilisant la suprématie de l'abeille mellifère ont été proposées (Rozen \& McGinley 1974; Michener \& Greenberg 1980; Michener 1981; Radchenko \& Pesenko 1994; Alexander 1992; RoigAlsina \& Michener 1993; Alexander \& Michener 1995). Le point crucial de la phylogénie morphologique des abeilles se situe dans le balancement de la structure de la langue. En effet, la structure de la glosse des Colletidae a d'abord été considérée comme plésiomorphe (i.e. primitive), proche de la structure ancestrale des guêpes fouisseuses. Des études fines de la structure de cette glosse ont montré que la structure de la langue des Colletidae était bien différente de celle de Sphecidae. La langue bifide et courte des Colletidae serait plus probablement une convergence évolutive et non en une plésiomorphie.

L'ambiguïté a été levée très récemment par les nouveaux outils moléculaires (Danforth et al. 2006a, b). Danforth et ses collaborateurs ont démontré de manière convaincante la "modernité" des Colletidae (Figure 4). Cette nouvelle hypothèse révolutionne notre conception de l'évolution du groupe des abeilles. Premièrement, les abeilles à langue courte n'apparaissent plus comme ancêtres des abeilles à langue longue. Elles sont visiblement apparues dans un second temps. Deuxièmement, les Melittidae forment maintenant un groupe paraphylétique à la base du clade des abeilles. Comme la plupart des Melittidae sont oligolectiques (Michez et al. 2004, 2006, 2007a, b; Michez \& Patiny 2005, 2006), c'est-à-dire spécialisés dans la récolte de pollen sur un nombre limité taxons floraux, l'oligolectisme est probablement un caractère ancestral et le polylectisme un caractère dérivé. Par ailleurs, la diversité des Melittidae étant maximale en Afrique, l'origine des abeilles est probablement liée à ce continent. L'ancêtre des abeilles était donc probablement un spécialiste africain et pas un généraliste gondwanien comme présenté par Michener (2000) (Danforth 2006b).

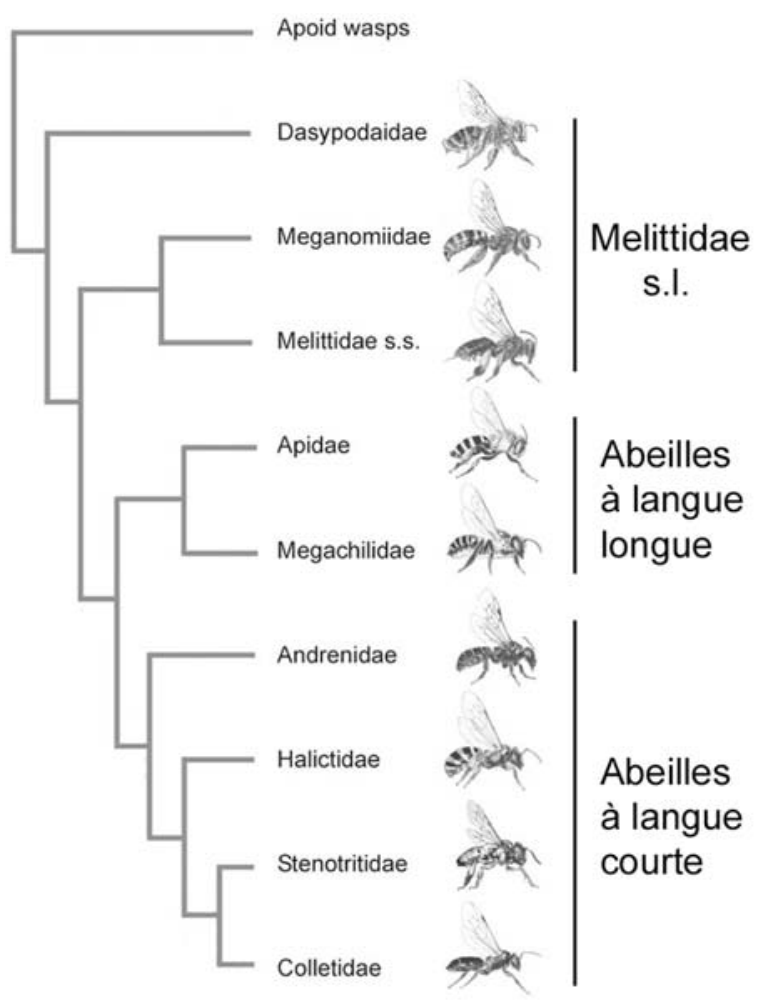

Figure 4. Phylogénie des Apoidea Apiformes basée sur la morphologie des adultes et le séquençage de 5 gènes (d'après Danforth et al. 2006).

L'hypothèse première de placer l'abeille mellifère au "sommet de l'évolution" des abeilles, et même des insectes, n'est certainement pas étrangère aux rapports privilégiés de cette espèce avec l'Homme. Le genre Apis est finalement peu diversifié (8 espèces contemporaines décrites) en regard du gigantesque genre Andrena (plus de 1200 espèces décrites), et le voilà maintenant relégué sur une branche parallèle de l'arbre évolutif de abeilles.

\section{Références bibliographiques}

d'Aguilar J, 2006. Histoire de l'entomologie. Delachaux et Niestlé, Paris, $224 \mathrm{p}$.

Alexander BA, 1992. An exploratory analysis of cladistic relationships within the superfamily Apoidea, with special reference to sphecid wasps (Hymenoptera). Fournal of Hymenoptera Research 1: 25-61.

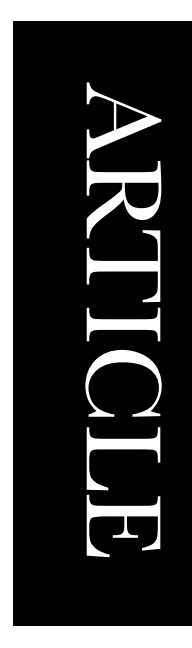


Alexander BA \& Michener CD, 1995. Phylogenetic studies of the families of short-tongued bees (Hymenoptera : Apoidea). The University of Kansas Science Bulletin 55: 377 - 424.

Danforth BN, Fang J \& Sipes SD, 2006a. Analysis of familylevel relationships in bees (Hymenoptera: Apiformes) using $28 \mathrm{~S}$ and two previously unexplored nuclear genes: CAD and RNA polymerase II. Molecular Phylogenetics and Evolution 39: 358-372.

Danforth BN, Sipes SD, Fang J \& Brady SG, 2006b. The history of early bee diversification based on five genes plus morphology. Proceedings of the National Academy of Sciences of the United States of America 103: 15118-15123.

Engel MS, 2005. Family-Group Names for Bees (Hymenoptera: Apoidea). American Museum Novitates 3476: 1-33.

Gould SJ, 1996. L'éventail du vivant. Seuil, Paris, 320p.

Kirby W, 1802. Monographia Apum Angliae. privately published, Ipswich, vol. 1 : 258p., vol. 2 : 388p.

Latreille PA, 1802. Histoire naturelle générale et particulière des Crustacées et des Insectes. Dufart, Paris, 467p.

Maeterlinck M, 1901. La vie des abeilles. Editions Tansatlantiques, Laval, 187p.

Michener CD, 1944. Comparative external morphology, phylogeny, and classification of the bees (Hymenoptera). Bulletin of the American Museum of Natural History 82: 1-326.

Michener CD, 1981. Classification of the bee family Melittidae with a review of species of Meganomiinae. Contribution of the American Entomological Institute 18: 1-135.

Michener CD, 2000. The bees of the world. The Johns Hopkins University Press, Baltimore, 913 p.

Michener CD, 2006. The Professional Development of an Entomologist. Annual Review of Entomology 52: 1-15.

Michener GD \& Greenberg $\mathbf{L}, \mathbf{1 9 8 0}$. Ctenoplectridae and the origin of long-tongued bees. Zoological Journal of the Linnean Society 69: 183-203.

Michez D, Else GR \& Roberts SPM, 2007. Biogeography, floral choices and re-description of Promelitta alboclypeata (Friese 1900) (Hymenoptera, Apoidea, Melittidae). African Entomology 15: sous presse.
Michez D, Eardley CD, Kuhlmann M \& Patiny S, 2007. Monographic revision of the southern-african bee genus Capicola (Hymenoptera: Apoidea: Melittidae). European fournal of Entomology: sous presse.

Michez D \& Patiny S, 2005. World revision of the oil-collecting bee genus Macropis Panzer 1809 (Hymenoptera, Apoidea, Melittidae) with a description of a new species from Laos. Annales de la Société entomologique de France (n. s.) 41: 15-28.

Michez D \& Patiny S, 2006. Review of the bee genus Eremaphanta Popov 1940 (Hymenoptera: Melittidae), with the description of a new species. Zootaxa 1148: 47-68.

Michez D, Patiny S, Rasmont P \& Vereecken N, 2006. Phylogeny and floral choices inheritance in bees. Scientific proceeding of IUSSI Congress XV, July 1 - Augustus 4, Washington D.C. (USA): 41-42.

Michez D, Terzo M \& Rasmont P, 2004. Phylogénie, biogéographie et choix floraux des abeilles oligolectiques du genre Dasypoda Latreille 1802 (Hymenoptera, Apoidea, Melittidae). Annales de la Société entomologique de France (n. s.) 40: 421-435.

Radchenko VG \& Pesenko YA, 1994. Biology of bees (Hymenoptera, Apoidea). Russian Academy of Sciences, Zoological Institute, St. Petersburg, $331 \mathrm{p}$.

Roig-Alsina A \& Michener CD, 1993. Studies of the phylogeny and classification of long-tongued bees (Hymenoptera: Apoidea). The University of Kansas Science Bulletin 55: 123-173.

Rozen JG \& Mcgingley RJ, 1974. Phylogeny and systematics of Melittidae based on the mature larvae (Insecta, Hymenoptera, Apoidea). American Museum Novitates 2545: 1-31.

Schenk A, 1860. Verzeichniss der nassauischen Hymenoptera aculeata mit Hinzufügung der überigen dem Verfasser bekannt gewordenen deutschen Arten. Stettiner Entomologische Zeitung 21: 132-157; 417-419.

Thomson CG, 1872. Skandinaviens Hymenoptera. Berling, Lund, 286p.

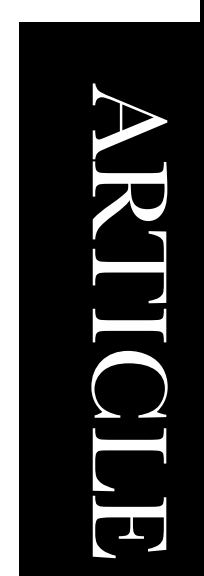

